\title{
Does torque minimization yield a stable human grasp?
}

\author{
Gabriel Baud-Bovy ${ }^{1}$, Domenico Prattichizzo ${ }^{2}$, and Nadia Brogi ${ }^{2}$ \\ 1 San-Raffaele Vita-Salute University, Milan, Italy \\ 2 Dipartimento di Ingegneria dell'Informazione, University of Siena, Italy
}

\begin{abstract}
In this paper, we present a study of the human grasp conducted with a mathematical formalism that has been developed in robotics during the last two decades. The main objective of this study is to assess the extent to which the structure of the hand is adapted to its grasping function by identify the conditions under which a model minimizing energy expenditure predicts a stable grasp. The idea is that the human hand, as the result of evolutionary pressure, must be designed in such a way that it can grasp objects with minimum effort. To test this hyptohesis, we defined a cost function minimizing the weighted norm of the joint torque vector of a simple biomechanical model of the hand. The contact forces predicted by the model are then compared to the ones observed in a experimental study of the human tripod grasp. The results indicate that this cost function can predict a stable grasp when the external force is zero. A possible interpretation of this result is that the external force represents an unknown that cannot be taken into account by an evolutionary process.
\end{abstract}

"The coordination of a movement is the process of mastering redundant degrees of freedom of the moving organ, in other words its conversion to a controllable system" (Bernstein, [6], p. 127).

\section{Introduction}

Research in robotic hands during the last two decades has led to the development of a mature theoretical framework that can be used to analyze the human grasp. In robotics, the theoretical foundations of grasping were established in one of the first works dealing with this subject [24]. An important result of this early work is that the task constraints do not suffice in general to specify fully the contact forces, and that the amount of under-determinacy increased with the number of fingers involved in the grasp [29]. For the motor control scientist, the study of multi-finger grasps represents an opportunity to investigate how redundant degrees of freedom are transformed into a controllable system [3,9,12,39,32] and, insofar, to address Bernstein's degrees of freedom problem [6].

Optimal control is a very general approach to identify a unique solution for under-determined problems in robotic and human studies alike [14]. The gist of the approach is to define a cost (or objective) function over the set of possible solutions and select the one that minimizes it. Numerous human studies have shown that the motor system minimizes jerk [13], torques [31], torque changes [35], noise [15], or angular displacement [5] depending on the context.

Minimizing energy expenditure and maximizing grasp stability are two important objectives in grasping [7,29,38,34]. These two objectives cannot, however, be always 
simultaneously achieved. In the pinch grasp for example, the only way to avoid contact slippage is to squeeze the object more (see [18] for a review of the pinch grasp in humans). In contrast, the additional degrees of freedom of the internal forces of multi-finger grasps allow one to increase grasp stability by varying the direction of the contact forces without increasing their magnitude.

In [3], the authors examined whether the direction of the contact forces maximized the contact stability of the tripod grasp. This study showed that stability played an important role but that the choice of the contact forces was sub-optimal from this point of view. This observation led to the formulation of a hierarchical control scheme based on the notion of virtual finger.

The present study investigates the possibility that the contact forces observed in the human tripod grasp minimize energy expenditure. The study addresses the question of whether the human hand, as the result of evolutionary pressure, is designed in such a way that it can grasp with minimum effort. In other words, this study is an attempt to assess the extent to which the hand structure is adapted to its grasping function. To answer this question, the cost function must exclude any reference to the contact constraints, that is, the model must allow the contact forces predicted by the model to violate contact constraints if this is more economical in terms of energy expenditure. From a methodological point of view, this study does not attempt to identify the best cost function to predict human grasps. Instead, it posits a particular cost function - one that minimizes the weighted norm of the joint torques - and examines the goodness of its predictions by comparing them to stable grasps that were observed in a previous study [3] conducted with humans.

Obviously, there are many ways to define energy expenditure in grasping. In this study, we attempted to evaluate the energetic cost of grasping in terms of joint torques and muscle activity rather than in terms of magnitude of the contact forces. For this reason, the minimum torque cost function includes a biomechanical model of the hand that relates contact forces to joint torques and other biomechanical factors such as muscle strenght and the moment arms of the tendons.

In Section 2, we introduce the formal framework required to define the minimum torque cost function (see Section 2.6). In Section 3, we summarize the results of an experimental study on the human tripod grasp [3]. These results are used in Section 4 as a benchmark to test the predictions of the minimum torque model. The implications and limits of our approach are discussed in Section 5.

\section{The minimum torque model}

In this section, we introduce the theoretical framework necessary to define the minimum torque cost function (see 2.6). We introduce first some well known concepts in robotics such as the soft and hard finger contact models (2.1), and two important matrices, the grasp matrix (2.2) and the hand Jacobian (2.5) [24,27]. Internal forces (2.3) and contact constraints (2.4) are discussed in the context of the tripod grasp. 

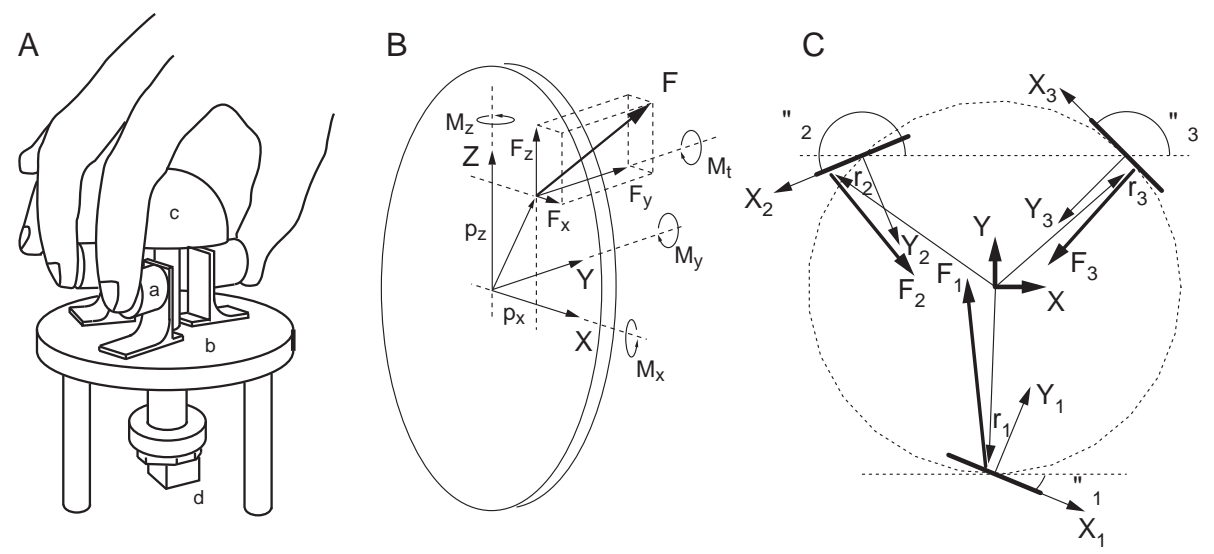

Fig. 1. $A$ : The tripod grasp. $B$ : The contact force $\mathbf{F}=\left(F_{x}, F_{y}, F_{z}\right)$ and normal torque $M_{t}$ is applied at the center of pressure $\mathbf{p}=\left(p_{x}, p_{y}\right)$ of the contact surface. The origin of the local coordinate system associated to each contact surface coincides with its center. $C$ : Contact forces $\mathbf{F}_{i}$ in the plane defined by the contact points $r_{i}$. The short thick lines indicate the position and orientation of the contact surfaces (adapted from [3]).

\subsection{Contact model}

Several models can be used to describe the contact interactions between the object and the finger $[30,10]$. In the soft-finger contact model, the mechanical interaction taking place between a finger and the object is modeled by a force $\mathbf{F}_{i}=$ $\left[F_{x i}, F_{y i}, F_{z i}\right]^{T}$ applied at the center of pressure $\mathbf{r}_{i}$ and a torque $\mathbf{M}_{i}=\left[0, M_{t}, 0\right]^{T}$, about the normal $\mathbf{n}_{i}$ passing through the center of pressure and perpendicular to the surface of the object (see Fig. 1). In the hard-finger contact model, the normal torques are zero.

In this study, all simulations were conducted using the hard finger model. While less realistic than the soft-finger contact model, the use of the hard-finger model in the context of this study is justified by the observation that the normal torques $M_{i}$ 's do not contribute to the horizontal components of the contact forces when the contact surfaces are vertically oriented. In fact, the normal torques can only redistribute the load (vertical components of the contact forces) across fingers [4].

In what follows the grasp model is introduced for the grasping configuration described in Fig. 1: the tripod grasp. The grasp consists of a human hand grasping an object through three hard-finger contact points $\mathbf{r}_{1}, \mathbf{r}_{2}$ and $\mathbf{r}_{3}$ corresponding to the thumb, the index and middle fingers, respectively. 


\subsection{Grasp matrix}

In this section, we define the grasp matrix that relates the contact forces to the net force. Let $\mathbf{t}$ be the 9 -elements vector obtained by grouping the contact forces at the three contact points $\mathbf{r}_{i}$ of the tripod grasp (Fig. 1),

$$
\mathbf{t}=\left[\mathbf{F}_{1}, \mathbf{F}_{2}, \mathbf{F}_{3}\right]^{T}
$$

and let $\mathbf{w}$ be the net wrench, consisting of a net force and a net torque, applied to the object. The grasp matrix represents the mapping between the contact forces and torques $\mathbf{t}$ and the space of wrenches $\mathbf{w}$

$$
\mathbf{w}=\mathbf{G t}
$$

The grasp matrix is obtained from the complete grasp matrix $\tilde{\mathbf{G}}$ and the contact selection matrix $\mathbf{H}[28]$.

The complete grasp matrix $\tilde{\mathbf{G}}$ is a $6 \times 12$-matrix

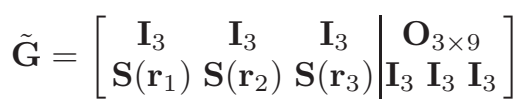

where $\mathbf{S}\left(\mathbf{r}_{i}\right)$ is the cross-product matrix for the contact point $\mathbf{r}_{i}$, i.e. the skewsymmetric matrix such that $\mathbf{S}\left(\mathbf{r}_{i}\right) \mathbf{p}_{i}=\mathbf{r}_{i} \times \mathbf{p}_{i}$.

The complete grasp matrix models the contact force interaction as if the contact surfaces of the object and of the fingers were glued. In this case contact forces and torques can be exchanged along and about all directions between the object and the fingers at the contact points.

In order to model other types of contact interactions, in [30] the contact selection matrix $\mathbf{H}$ is introduced. This matrix selects only directions that are constrained during the contact interactions and allow to exchange forces and torques between the object and the hand. For the hard-finger contact model only forces can be exchanged and the selection matrix is

$$
\mathbf{H}=\left(\begin{array}{lll}
\mathbf{I}_{3} & \mathbf{O}_{3} & \mathbf{O}_{3} \\
\mathbf{O}_{3} & \mathbf{I}_{3} & \mathbf{O}_{3} \\
\mathbf{O}_{3} & \mathbf{O}_{3} & \mathbf{I}_{3}
\end{array} \mid \mathbf{O}_{9}\right)
$$

Finally, the grasp matrix turns to be

$$
\mathbf{G}=\tilde{\mathbf{G}} \mathbf{H}^{T}=\left[\begin{array}{ccc}
\mathbf{I}_{3} & \mathbf{I}_{3} & \mathbf{I}_{3} \\
\mathbf{S}\left(\mathbf{r}_{1}\right) & \mathbf{S}\left(\mathbf{r}_{2}\right) & \mathbf{S}\left(\mathbf{r}_{3}\right)
\end{array}\right]
$$

\subsection{Internal forces}

When holding an object immobile, the finger forces and the external wrenches are in equilibrium. The corresponding equilibrium condition is simply obtained as

$$
\mathbf{w}_{\text {ext }}=-\mathbf{G t}
$$




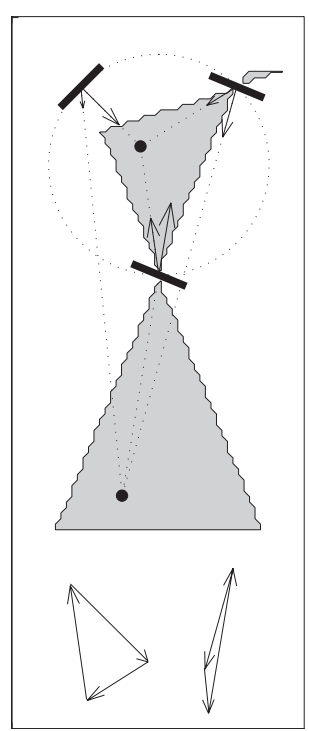

Fig. 2. Example of contact forces in the tripod grasp. Both sets of contact forces result in a zero net force in the plane defined by the three contact points. When the net force is zero, the force directions intersect at a point called the force focus (black dot) and the three contact forces drawn tail-to-toe form a triangle called the force triangle. The hatched area corresponds to the set of all valid force focus positions that correspond to forces satisfying both the friction and unilateral constraints.

where $\mathbf{w}_{e x t}$ is the external wrench applied to the object including the gravity force. Equations (5) also holds true for non equilibrium conditions provided that the external wrench accounts also for object dynamics.

In general, the problem of finding the finger forces that match a given external wrench is under-determined. For example, Fig. 2 shows two sets of contact forces in the tripod grasp that produce a zero net wrench in the plane defined by the three contact points. The general solution to the equilibrium equation (5) is

$$
\mathbf{t}=-\mathbf{G}_{R} \mathbf{w}_{e x t}+\mathbf{E y}=\mathbf{t}_{v}+\mathbf{t}_{h y}
$$

where $\mathbf{G}_{R}$ is any right inverse of the grasp matrix $\mathbf{G}$ and $\mathbf{E}$ is a basis of the nullspace of the grasp matrix defining the subspace of internal forces, i.e. those self-balanced contact forces such that $\mathbf{G E y}=\mathbf{0}$. In robotic grasp literature, forces $\mathbf{t}_{h y}=\mathbf{E y} \in \operatorname{ker}(\mathbf{G})$ belonging to the nullspace of the grasp matrix represent the internal forces and play a fundamental role in grasp contact stability (slippage avoidance) [30]. The other component of the solution $\mathbf{t}_{v}$ is usually referred to as the manipulating component of the contact forces [38].

In the tripod grasp with the hard-finger model for the three contacts, the internal forces enjoy the following properties: 
1. The null space of the grasp matrix has dimension three $(\operatorname{dim}(\operatorname{ker}(\mathbf{G}))=$ $\operatorname{rank}(E)=3$ ), there are therefore three unconstrained DoFs represented by the vector $\mathbf{y} \in \Re^{3}$ in the solution (6) to (5);

2. The three internal forces components $\mathbf{F}_{h_{i}} \in \Re^{3}$, where $\mathbf{t}_{h y}=\left[\mathbf{F}_{h_{1}}, \mathbf{F}_{h_{2}}, \mathbf{F}_{h_{3}}\right]^{T}=$ Ey $\in \Re^{9}$, lie in the plane defined by the three contact points $\mathbf{r}_{i}$.

The three-dimensional internal forces subspace admits other parametrizations than the vector $\mathbf{y}$. In particular, [38] defines the force triangle as the triangle of internal forces $\mathbf{F}_{h_{i}}$ 's at the three contact points $(i=1, \ldots, 3)$ when drawn tip-totail $\left(\sum \mathbf{F}_{h_{i}}=\mathbf{0}\right)$ and the force focus as the unique intersection point of the three directions of the internal forces. In [3], the perimeter of the force triangle is referred to as the grip force, $F_{\text {grip }}$. The position of the force focus in the plane defined by the three contact points (see Fig. 2) and the grip force can be used as a new triple of parameters for the internal force vector $\mathbf{t}_{h y}$. The position of the force focus depends only on the direction of the contact forces while the grip force depends only on their magnitude. Therefore, one can argue that grasp stability is mainly related to the position of the force focus while energy expenditure is related to the grip force in the tripod grasp.

\subsection{Contact constraints}

The contact forces must satisfy the contact constraints that include both the unilateral and frictional constraints at all contacts $i=1, \ldots, 3$. Assume that all the contact normals are oriented towards the center of the object, or in other terms inside the object. The unilateral constraints are formalized as

$$
\mathbf{n}_{i}^{T} \mathbf{F}_{i} \geqslant 0
$$

and state that a finger can only push and not pull the contact surface (i.e., the normal component of the contact must be positive). The frictional constraints state that the contact force must belong to the friction cone

$$
\left\|\left(I-\mathbf{n}_{i} \mathbf{n}_{i}^{T}\right) \mathbf{F}_{i}\right\| \leqslant \mu \mathbf{F}_{i}^{T} \mathbf{n}_{i}
$$

where $\mu$ is the linear coefficient of friction defining the minimum normal force required to avoid a slip of the finger in presence of a tangential force.

Contact grasp stability is an important property of the grasp that refers to its ability to resist external wrenches, in agreement with (5), without violating the contact constraints (7) and (8). In other words, only the contact forces defined in (6) that satisfy the contact constraints yield a stable grasp.

For the tripod grasp, the internal forces lie in the plane defined by the three contact points and can be parameterized by the force focus and the grip force. Fig. 3 shows the regions of the plane where the force focus must be located to satisfy the contact constraints under the assumption that the net force is zero. The dark grey areas represent the intersection of the light and medium gray area and identify positions of the force focus that satisfy both unilateral and frictional constraints. The light and medium gray areas indicate the regions that satisfy only the unilateral and 

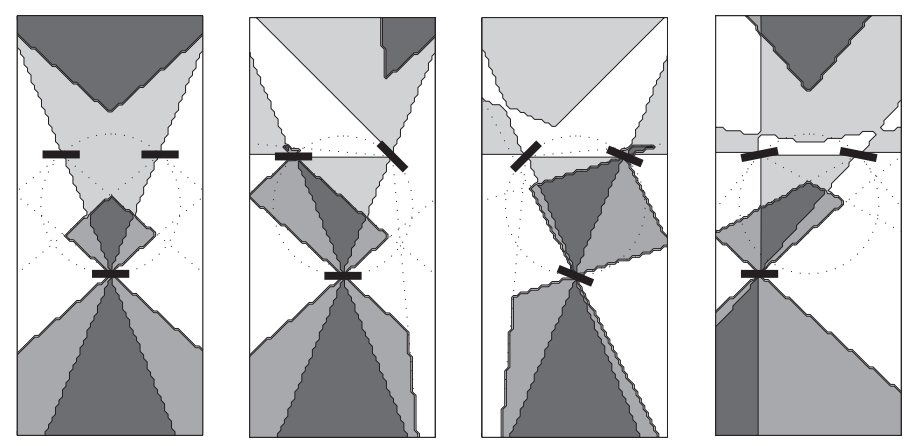

Fig. 3. Valid force focus positions for various positions and orientation of the contact surfaces. The positions of the force focuses that satisfies both the squeezing and frictional constraints are indicated in dark gray. Force focus positions that satisfy only frictional or squeezing constraints are indicated in medium and light grey, respectively.

frictional constraints respectively. Geometrically, the medium gray areas correspond to the intersection of three double cones, each one being centered on a contact point and having an aperture of $\alpha=\tan ^{-1}(\mu)$. Note that the contact grasp stability depends only on the position of the force focus and not on the grip force.

The position of the force focus is similarly constrained when the external force is orthogonal to the plane defined by the contact points. In this case however, the aperture of the double cones that underlie the medium grey areas in Fig. 3 depends on the relative magnitude of the external and grip force. For a given level of grip force, the aperture of the cones will decrease until it might not be possible to achieve a stable grasp. In this case, it is necessary to increase the grip force like in the pinch grasp to avoid a slip of the object.

\subsection{Hand Jacobian}

In this section, we describe the hand Jacobian that relates the joint torques of the hand to the contact forces applied on the object by the fingers. For the tripod grasp in Fig. 1-A, determining the hand Jacobian involves the study of the biomechanics of the hand $[20,22,26,19]$.

The Jacobian transpose $\mathbf{J}^{T}$ of the tripod grasp relates the overall contact force vector $\mathbf{t}$ (1) with the overall joint torque vector $\tau$ of the three fingers involved in the grasp

$$
\tau=\mathbf{J}^{T} \mathbf{t}
$$

The complete torque vector $\tau^{T}=\left[\tau_{1}^{T}, \tau_{2}^{T}, \tau_{3}^{T}\right]$ is obtained by grouping the vector of torques of the thumb $\tau_{1}$, index $\tau_{2}$ and middle $\tau_{3}$ fingers.

The model of the hand includes only the fingers involved in the tripod grasp, i.e. the thumb, index and middle fingers. All fingers have four DoFs, yielding a total of twelve DoFs. The MCP joint of the index and middle fingers, as well as the TM joint 


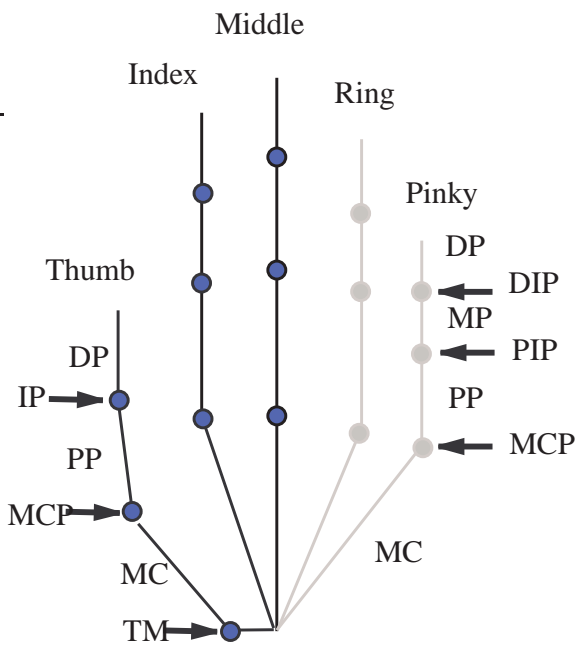

Fig. 4. Schematic representation of the hand model. Joint names: metacarpophalangeal (MCP), proximal interphalangeal (PIP), distal interphalangeal (DIP), trapeziometacarpal (TM), joint interphalangeal (IP). Link names: metacarpal (MC), proximal phalanx (PP), middle phalanx (MP), and distal phalanx (DP).

of the thumb, have two DoFs each (adduction/abduction and flexion/extension). The PIP and DIP joints of the index and middle fingers, as well as the MCP and IP joints of the thumb have one DoF each (see Fig. 4).

Table 1 and Fig. 5 show the Denavit-Hartenberg notation used to define the hand Jacobian corresponding to the model of the hand.

Index finger
\begin{tabular}{|c|c|c|c|c|}
\hline link & $a_{i}$ & $\alpha_{i}$ & $d_{i}$ & $\theta_{i}$ \\
\hline 1 & $m c_{i}$ & 0 & 0 & $\theta_{i n}$ \\
2 & 0 & $-90^{\circ}$ & 0 & $\theta_{1}$ \\
3 & $p p_{i}$ & 0 & 0 & $\theta_{2}$ \\
4 & $m p_{i}$ & 0 & 0 & $\theta_{3}$ \\
5 & $d p_{i}$ & 0 & 0 & $\theta_{4}$ \\
\hline
\end{tabular}

Middle finger
\begin{tabular}{|c|c|c|c|c|}
\hline link & $a_{i}$ & $\alpha_{i}$ & $d_{i}$ & $\theta_{i}$ \\
\hline 1 & $m c_{m}$ & 0 & 0 & 0 \\
2 & 0 & $-90^{\circ}$ & 0 & $\theta_{1}$ \\
3 & $p p_{m}$ & 0 & 0 & $\theta_{2}$ \\
4 & $m p_{m}$ & 0 & 0 & $\theta_{3}$ \\
5 & $d p_{m}$ & 0 & 0 & $\theta_{4}$ \\
\hline
\end{tabular}

Thumb
\begin{tabular}{|c|c|c|c|c|}
\hline link & $a_{i}$ & $\alpha_{i}$ & $d_{i}$ & $\theta_{i}$ \\
\hline 1 & $a_{1}$ & $75^{\circ}$ & $d_{1}$ & $\theta_{t}$ \\
2 & 0 & $90^{\circ}$ & 0 & $\theta_{1}$ \\
3 & $m c_{t}$ & $90^{\circ}$ & 0 & $\theta_{2}$ \\
4 & $p p_{t}$ & 0 & 0 & $\theta_{3}$ \\
5 & $d p_{t}$ & 0 & 0 & $\theta_{4}$ \\
\hline
\end{tabular}

Table 1. The Denavit-Hartenberg parameters of the biomechanical model of the hand.

Having computed the kinematic model of the hand, the hand Jacobian is simply obtained from $[28,29]$. 


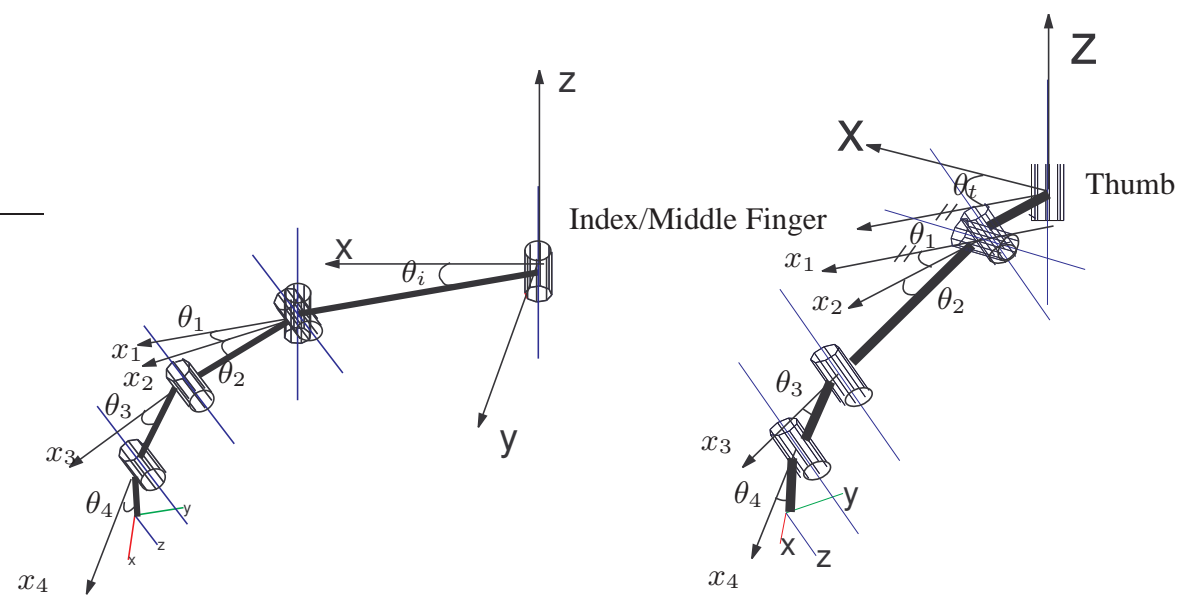

Fig. 5. Kinematic model of the hand (only the thumb, index and middle fingers are included in the model).

Similar to the computation of the grasp matrix, we first evaluate the complete hand Jacobian $\tilde{\mathbf{J}}$ and then multiply the result by the selection matrix $\mathbf{H}$ to get the hand Jacobian

$$
\mathbf{J}=\mathbf{H} \tilde{\mathbf{J}}=\left(\begin{array}{ccccc}
\mathbf{D}_{\mathbf{1}, \mathbf{1}}{ }^{T} & \mathbf{D}_{\mathbf{1}, \mathbf{2}}{ }^{T} & \ldots & \mathbf{D}_{\mathbf{1}, \mathbf{1 2}}{ }^{T} \\
\mathbf{D}_{\mathbf{2}, \mathbf{1}}{ }^{T} & \mathbf{D}_{\mathbf{2}, \mathbf{2}}{ }^{T} & \ldots & \mathbf{D}_{\mathbf{2}, \mathbf{1 2}}{ }^{T} \\
\vdots & \vdots & \ddots & \vdots \\
\mathbf{D}_{\mathbf{n}, \mathbf{1}}{ }^{T} & \mathbf{D}_{\mathbf{n}, \mathbf{2}}{ }^{T} & \ldots & \mathbf{D}_{\mathbf{n}, \mathbf{1 2}}{ }^{T}
\end{array}\right)
$$

being $\mathbf{D}_{i, j}=\mathbf{S}\left(\mathbf{c}_{i}-\mathbf{o}_{j}\right) \quad\left(\mathbf{D}_{i, j}=\mathbf{O}_{1 \times 3}\right)$ if the $i$-th contact force affects (does not affect) the $j$-th joint where $\mathbf{c}_{i}$ is the vector of the $i$-th contact point and $\mathbf{o}_{j}$ the center of the joint.

The Jacobian matrix plays a key role to identify the internal forces that can be controlled by the hand. The importance of controllability of internal forces in grasping was put into evidence in a quasi-static setting in [7] and in a dynamic context in $[27,29]$. These works have shown that the controllable internal forces belong to the subspace obtained by a weighted projection of the column space of the hand Jacobian $\mathbf{J}$ onto the nullspace of the grasp matrix $\mathbf{G}$

$$
\operatorname{span}\left(\left(\mathbf{I}-\mathbf{G}_{\overline{\mathbf{K}}}^{+} \mathbf{G}\right) \overline{\mathbf{K}} \mathbf{J}\right)
$$

where $\mathbf{G}_{\overline{\mathbf{K}}}^{+}$is the $\overline{\mathbf{K}}$-weighted pseudoinverse of $\mathbf{G}$, and $\overline{\mathbf{K}}_{c}^{-1}=\mathbf{K}^{-1}+\mathbf{J R}_{q}^{-1} \mathbf{J}^{T}$ is the equivalent stiffness matrix including the effect of proportional control $\mathbf{R}_{q}$ on joint positions and the contact stiffness $\mathbf{K}_{c}$ [11].

For the tripod grasp, it can be shown that the subspace of reachable internal forces is equal to the nullspace of the grasp matrix G. In other terms, in the tripod grasp all the internal forces result to be controllable by the human hand kinematics. 


\subsection{The minimum torque cost function}

This section is devoted to the development of a cost function able to predict the contact forces in the human tripod grasp.

At first sight, the grip force, i.e., the sum of the intensities of the three contact forces in the tripod grasp (see Section 2.3), appears as a plausible candidate to measure energy expenditure in the grasp. The main problem of this approach is that it neglects important biomechanical factors such as muscle strength or the moment arm of the tendons that might have a strong impact on the actual energetic cost required to produce contact forces.

To measure energy expenditure, we propose to use a well-known cost function in the robotics literature [25] that is the weighted norm of the hand joint torques:

$$
C(\mathbf{y})=\|\mathbf{K} \tau(\mathbf{y})\|
$$

where $\tau$ is the $12 \times 1$ vector of torques in (9) and $\mathbf{K}$ is the $12 \times 12$ diagonal matrix of weights that represent biomechanical factors involved in the generation of the contact forces. For a given external wrench, it follows from (6) that the joint torque vector is uniquely determined by the three-dimensional vector $\mathbf{y}$ parameterizing the internal force vector

$$
\tau(\mathbf{y})=\mathbf{J}^{T} \mathbf{t}(\mathbf{y})
$$

where $t$ is the vector of all contact forces (1). Note that this cost function does not consider the orientation of the contact surfaces and, therefore, cannot take into account the frictional or unilateral constraints.

The proposed cost function will be used in the next sections to predict the human grasp. Two minimization problems are considered.

The first one is unconstrained and minimizes the cost function (11) over the subspace of internal forces that, for the tripod grasp, is a three dimensional space that can be parameterized either by the vector $\mathbf{y}$ or by the force focus position and grip force $F_{\text {grip }}$ (see Section 2.3).

The second minimization problem is a constrained problem and corresponds to minimize the cost function (11) under the constraint that the grip force is constant

$$
F_{\text {grip }}=\mathrm{const}
$$

This constraint allows to prevent that the optimal solution is the zero torque vector when the external force is zero.

It is an easy matter to verify that, in case of zero external wrenches on the objects, the level of the constant grip force in (13) does not affect the optimal solution. On the other hand, in case of non zero external wrenches on the objects, the minimizing cost function (11) under constraint (13) provides a solution that depends on the value of the constant used in constraint (13). 


\section{Experiments on human tripod grasp}

The results on the human tripod grasp presented in [3] are summarized here for the reader's convenience. These results are used as a benchmark to test the predictions of the minimum torque model in the next section.

In [3], subjects grasped an object with the thumb, index and middle fingers as described in Fig. 1. The position and orientation of the contact surfaces varied across experimental conditions, yielding a total of forty seven distinct grasping configurations. The contact forces were measured by three force transducers mounted under the three contact surfaces. The task consisted simply in grasping, lifting and holding the object immobile in the air. The movement of the object was monitored by an electromagnetic motion capture system. Visual feedback was provided to ensure that the plane defined by the three contact points was horizontal. The net wrench was therefore zero in the horizontal plane and equal to the object's weight $m g$ in the

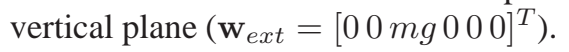

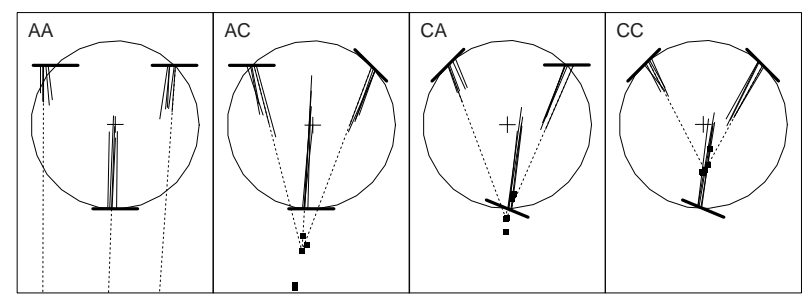

Fig. 6. Each panel represents a top view of the contact forces (thin solid lines) in the horizontal plane during five trials executed in the same condition by one subject (adapted from [3]). The short thick lines represent the contact surfaces. For each trial, the black dot denotes the position of the force focus (i.e., the intersection between the directions of the three contact forces). Labels AA, AC, CA, CC identify the orientation of the index and middle finger contact surfaces.

Fig. 6 plots the the horizontal component of the contact forces applied by one participant during the holding phase of a lift repeated five times. Each panel corresponds to a different experimental condition. The orientation of the index and middle finger contact surfaces are different in each panel. While the direction of the index and middle finger contact forces varied across panels, the direction of the thumb force remained invariant.

This figure illustrates the main empirical finding of [3], which was the observation that the direction of the force exerted by the thumb did not depend on the orientation of the surfaces touched by the two opposing fingers.

Fig. 6 also shows that the directions of the three contact forces intersect at a single point, the force focus, as expected. It can be shown that the manipulating component $\mathbf{t}_{v}=-\mathbf{G}_{R} \mathbf{w}_{\text {ext }}$ of the general solution (6) is zero in the horizontal 
plane when the forces are self-balanced in the horizontal plane. In this case the force vector $\mathbf{F}_{i}$ at the contact $i$ (Fig. 1) can be decomposed as follows

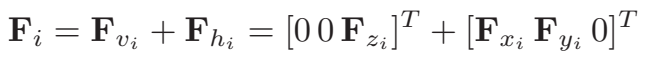

Therefore, the distinction between internal force $\mathbf{F}_{h_{i}}=\left[\mathbf{F}_{x_{i}} \mathbf{F}_{y_{i}} 0\right]^{T}$ and contact forces $\mathbf{F}_{i}$ in the horizontal plane is immaterial and the horizontal components $\mathbf{F}_{x_{i}}$ and $\mathbf{F}_{y_{i}}$ of the contact forces satisfy all the properties of the internal forces described in Section 2.3. In particular, the horizontal components of the contact forces, as the internal forces, intersect at a single point, the force focus, and form a force triangle.

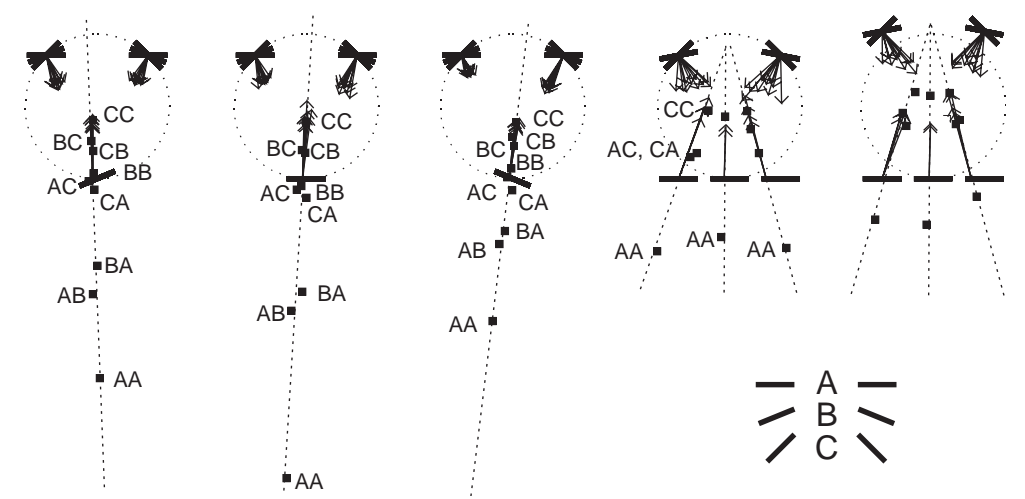

Fig. 7. Summary of the main findings of [3]. Black dots represent the average position of the force focuses for each experimental conditions. Labels A, B and C refer the orientations of the index and middle finger contact surfaces.

Fig. 7 summarizes the results of [3]. It shows the average position of the force focuses observed in forty seven different experimental conditions. The results for nine grasping configurations obtained by combining three possible orientations for the index and middle finger contact surfaces are represented in the first three panels (the labels next to the force focuses identify the corresponding grasping configurations). The orientation of the thumb contact surface is different in each one of these panels. The two last panels combine various orientations of the index and middle contact surfaces with three different thumb positions, yielding a total of twenty additional grasping configurations. The main difference between the two last panels on the right lies in the distance between the thumb and the opposing fingers.

As in Fig. 6, the direction of the thumb force did not depend on the orientation of the index and middle finger contact surfaces. This figure also shows that the index and middle finger forces are directed toward the center of the object when the index and middle contact surfaces also face the center of the object (condition CC in Figs. 6 and 7) and away from it when the two contact surfaces are more parallel (e.g., condition AA). These changes in the direction of the index and middle finger contact forces improve the stability of the grasp by maintaining the direction of the contact forces close to the normals. 
Altogether, these observations led to the formulation of a hierarchical control scheme whereby the two fingers opposing the thumb act synergistically as a virtual finger placed at mid-distance between the two opposing fingers $[16,17]$. The position of the force focus along the line defined by the thumb and virtual finger positions would be determined by stability considerations (see [3] for more details).

\section{Model-based prediction of human tripod grasp}

In this section, we investigate whether the minimum torque cost function, proposed in Section 2.6, yields results that can be favorably compared to the experimental results of the previous section. It will be shown that the most striking result is the role played by the external force (i.e., gravity) in the model. The effects of of slightly modifying the hand posture or the weighting matrix $\mathbf{K}$ on the predictions are also investigated.

\subsection{The hand posture}

The minimum torque cost function (11) depends on the hand Jacobian $\mathbf{J}$ defined in Section 2.5, which is a function of fixed parameters of the hand such as the length of the finger phalanxes, and of the joint angles $\theta_{i}$ that define a particular hand posture.

To identify the parameters of the hand Jacobian, we took pictures of the hand of subjects grasping the object in each one of the experimental conditions presented in 3. The size of the phalanxes, position of the palm relative to the object, and interphalangeal (IP) angle of the thumb were measured on the pictures and used to solve the inverse kinematic problem for each finger separately.

For the thumb, knowledge of the position of the thumb tip relative to the palm and of the IP angle is sufficient to compute the value of the remaining three DoFs. For the index and middle fingers, we assumed that the following constraint between DIP and PIP joint angles of the index and middle finger holds [20,22]:

$$
\theta_{D I P}=\frac{2}{3} \theta_{P I P}
$$

The hand Jacobian $\mathbf{J}_{\text {obs }}$ was computed for each experimental condition separately.

We also computed an average hand posture, with the corresponding average hand Jacobian $\mathbf{J}_{a v}$, by solving the inverse kinematic problem for the average palm position (and thumb IP joint value) observed across all the experimental conditions presented in Section 3.

\subsection{Muscles and tendons}

The predictions of the minimum torque model also depend on the diagonal weighting matrix $\mathbf{K}$ in (11), which is used to take into account biomechanical factors such as muscle strength and the moment arm of the tendons. Two different weighting matrices $\mathbf{K}_{b}$ and $\mathbf{K}_{h}$ have been used to predict the human grasp (see Table 2). 


\begin{tabular}{|l||l|l|l||l|l|l|}
\hline \multicolumn{1}{|l||}{} & $\mathbf{K}_{b}$ & \multicolumn{3}{|l||}{$\mathbf{K}_{h}$} \\
\hline DoFs & thumb & index & middle & thumb & index & middle \\
1 & 0.3 & 7.3 & 7.3 & 0.1 & 4.0 & 4.0 \\
2 & 0.7 & 0.5 & 0.5 & 0.1 & 1.0 & 1.0 \\
3 & 0.7 & 1.0 & 1.0 & 0.5 & 2.0 & 2.0 \\
4 & 3.1 & 5.2 & 5.2 & 1.0 & 3.0 & 3.0 \\
\hline
\end{tabular}

Table 2. Weights representing elements of the diagonal matrix $\mathbf{K}$

The diagonal elements of matrix $\mathbf{K}_{b}$ were obtained by computing, for each finger joint $j$, the inverse of the moment $\operatorname{arm} m_{j}$ of the relevant tendon multiplied by the physiological cross-sectional area $\mathrm{PCSA}_{j}$

$$
k_{j}=\left(m_{j} \mathrm{PCSA}_{j}\right)^{-1}
$$

Values for the moment arms were taken from [33] for the thumb and from [2] for the index and middle fingers. Values for the PCSAs were taken from $[1,23,21]$. When more than one tendon or muscle acted on a joint, we assumed that all relevant muscle contributed equally and computed the average value of the corresponding coefficients. The matrix $\mathbf{K}_{h}$ in Table 2 is a slight modification of $\mathbf{K}_{b}$ that improves the results of the simulations (see Section 4).

Though our approach suffers from limitations discussed in Section 5, the two matrices in Table 2 are meaningful from a biomechanical point of view. In general, small weighting coefficients correspond to joints associated with stronger muscles. For example, the abduction/adduction movements of index and middle fingers are produced by the comparatively small intrinsic muscles of the hand. In contrast, the flexion/extension movement are produced by the larger extrinsic muscles in the forearm. No such a difference exist at the MP joint of the thumb where the difference between intrinsic and extrinsic muscles is less relevant. For all fingers, the cost for producing a given flexion/extension torque increases for mechanical reasons from the proximal to the distal joint.

\subsection{Model prediction}

The minimum torque model predicted highly unrealistic contact forces when the contact forces needed to balance the gravitational force. For example, Fig. 8-A shows the predicted forces (thin black line) obtained with the average hand posture $\mathbf{J}_{a v}$ and the biomechanical matrix $\mathbf{K}_{b}$. As expected, the contact forces form a force triangle and the directions of the three contact forces intersect at a single point the force focus - (see the small solid square close to the index finger in Fig. 8). These observations imply that the equilibrium conditions are satisfied. In contrast, the predicted contact forces violated both the frictional and unilateral constraints. As matter of fact, the position of force focus is clearly outside of the hatched area satisfying both constraints in Fig. 3. Note that the violation of the unilateral 

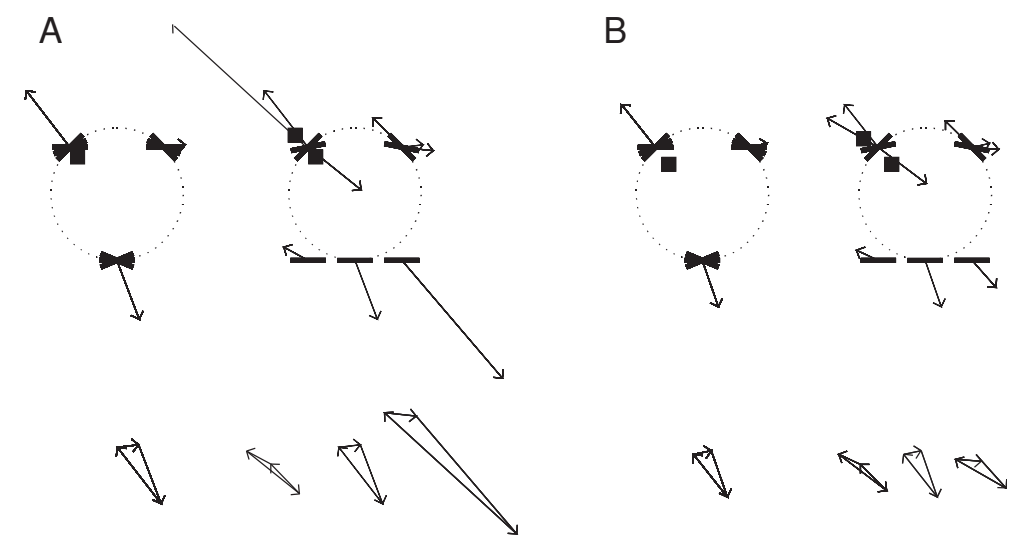

Fig. 8. Forces predicted by the minimum torque model with gravity (average hand posture $\mathbf{J}_{a v}$ and biomechanical weighting matrix $\mathbf{K}_{b}$ ). The force triangles are plotted below. The thumb position is shifted laterally in some panels (the short black lines represent the contact surfaces). A: Grip force unconstrained. B: Constrained grip force $\left(F_{\text {grip }}=5 \mathrm{~N}\right)$.

constraints implies that the fingers would not only slip but release the object in the real world.

The predictions of the minimum torque model do not depend on the orientation of the contact surfaces when using the average hand posture. For this reason, the model predicted the same contact forces for the twenty seven different combinations of contact surface orientations in the left panel of Fig. 8-A. Accordingly, the right panel of Fig. 8-A shows that the contact forces predicted by the model depended only on the thumb position. In Fig. 8-B, we constrained the grip force to $1 \mathrm{~N}$ (see eq. (13)) to examine the effect of the grip force on the predicted forces (see Section 2.6). Interestingly, we found that that the results improved considerably for larger grip forces $\left(F_{\text {grip }}>10 \mathrm{~N}\right)$. For value above $10 \mathrm{~N}$, the results did not differ significantly from the limit case (i.e., infinite large grip force) which is akin to assuming that the gravity is zero $\left(\mathbf{w}_{e x t}=\mathbf{0}\right)$.

Fig. 9-A shows the predicted forces in the limit case, i.e., in absence of gravity. The thumb and index forces are now pointing inward. Finally, by slightly modifying the matrix $\mathbf{K}$ from $\mathbf{K}_{b}$ to $\mathbf{K}_{h}$, it is possible to improve the results so that the predicted forces satisfy both the unilateral and frictional constraints whatever the thumb position (see Fig. 9-B). Like the experimental results (compare Fig. 9-B with Fig. 7), the thumb force is now directed toward a point at mid-distance between the index and middle finger.

So far, we have assumed that the hand posture remained fixed in all the experimental conditions and used the average hand Jacobian in the model. Actually, a detailed inspection of the hand posture across experiments conditions revealed subtle changes in the hand posture across experimental conditions (see Section 4.1). In principle, one could could adjust the hand posture as a function of the positions and 
A

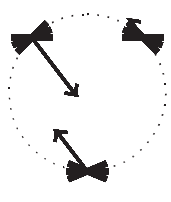

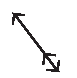

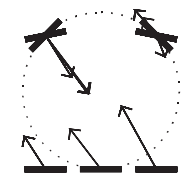

$\searrow \searrow \searrow$
B
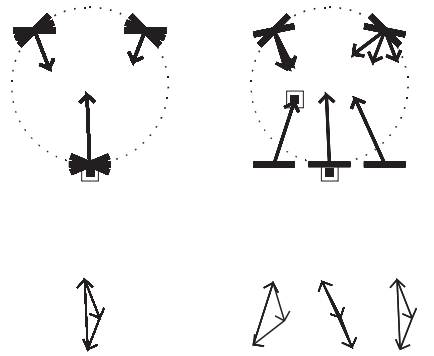

Fig. 9. Effect of matrix $\mathrm{K}$ on the predicted forces in absence of gravity (average hand posture, and constrained grip force). Same format as Fig. 8. A: Biomechanical weight matrix $\mathbf{K}_{b}$. B: Heuristic weight matrix $\mathbf{K}_{h}$.

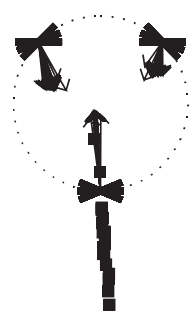

-
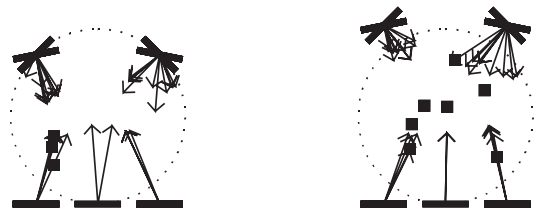

Fig. 10. Effect of hand posture on the predicted forces (no gravity, heuristic weight matrix $\mathbf{K}_{h}$, observed Jacobian, and constrained grip force). The solid square refer to the the position of the force focuses.

orientations of the contact surfaces to improve the stability of the grasp predicted by the minimum torque model.

Fig. 10 shows the contact forces predicted when using the observed hand postures instead of the average hand posture. The force focuses are now aligned along the direction of the thumb force as in Fig. 7.

To quantitavely assess the goodness of fit of the minimum torque model, we computed the average angular error

$$
\frac{1}{N} \sum \cos ^{-1} \frac{\mathbf{F} \hat{\mathbf{F}}}{|\mathbf{F}||\hat{\mathbf{F}}|}
$$

between the observed contact forces $\mathbf{F}$ in human tripod grasp, and the predicted contact forces $\hat{\mathbf{F}}$ across the $N=47$ experimental conditions considered in Section 3 .

Fig. 11 shows the goodness of the fit of the minimum torque model under the various assumptions discussed in this Section. The best results are clearly obtained with the adjusted weighting matrix $\mathbf{K}_{h}$ in absence of gravity. 


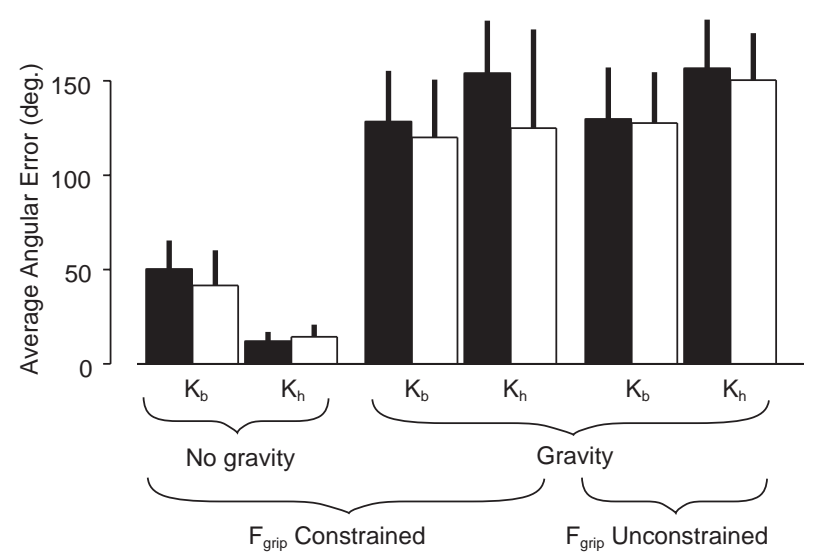

Fig. 11. Average angular errors between observed predicted contact forces (thin vertical lines represent SDs) over all experimental conditions considered in [3]. The solid and empty columns denote the results obtained with the average and observed hand postures respectively.

Regarding the hand posture, Fig. 11 shows the hand posture had a smaller effect on the goodness of the fit of the model than the external force or the weighting matrix $\mathbf{K}$. In particular, this figure shows the observed hand postures did not improve the results when the external force was zero and the heuristic weight matrix $\mathbf{K}_{h}$ was used. This observation indicates that the predicted force focus positions along thumb force direction in Fig. 10 differed from the one observed in the experiments (see Fig. 7). In other words, the variations in the hand postures observed in the experiments did not improve the stability of the grasps predicted by the model.

\section{Discussion}

In the Introduction, we mentioned the fact that the relationship between grasp stability and energy expenditure is not necessarily antagonistic for multi-finger grasps. For example, it is possible to increase the stability of the grasp without increasing the grip force in the tripod grasp. Since these two objectives do not necessarily conflict, the question of whether a stable grasp is economical and vice-versa is valid within the context of multi-fingered grasps. More generally, the question of whether the hand is adapted to its function, and has evolved so that it can grasp most objects in most circumstances in an economical way, also seems legitimate.

This study shows that minimizing the (appropriately weighted) joint torques can predict contact forces that satisfy the contact constraints. In other words, the most economical grasp is also a stable grasp under certain conditions that we will examine shortly. Such a result was not preordained because the minimum torque model did not represent purposefully the directions of the contact forces and the contact constraints.

Formally, the predictions of the minimum torque model depend on the hand Jacobian $\mathbf{J}$, on the joint weighting matrix $\mathbf{K}$ and on the external force $\mathbf{w}_{\text {ext }}$. While 
there is some flexibility at the level of the hand posture (i.e., joint angle) that can be used to grasp an object to balance an external force, the main parameters of the model (i.e., the position of the fingers relative to the palm, phalanx length etc) are fixed since they represent biomechanical features of the human hand.

To achieve the best results for the model prediction, it was necessary for the external force to be zero (no gravity). To understand this condition, it might be useful to recall that the hand cannot be modified to the peculiar circumstances in which the grasp takes place. Nor is there any reason to think that the hand minimize energy expenditure in a particular grasping situation, say when the object is held in such a way that the external force is orthogonal to the plane defined by the contact points. Therefore, it might be best to think of the external force - from the point of view of an evolutionary process - as an unknown and, in any way, uncontrollable factor that cannot be taken into consideration. Alternatively, one may think of this condition as a compromise, in some average sense, between all possible external forces.

Several caveats are nevertheless in order. First, the development of a biomechanical model of the hand is a complex endeavour [36]. Our approach to represent biomechanical factors such as the moment arm of the tendons and the muscle strength by the matrix $\mathbf{K}$ suffers from strong limitations. First and foremost, different muscles act on each joint. For example, extensor digitorum communis (EDC), the extensor indicis proprious (EIP), first palmar interosseous (PI) and first lumbrical (LUM) are part of the extensor mechanism of the index. Similarly, the flexor digitorum profundus (FDP), flexor digitorum superficialis (FPS) flex the index [37]. The mechanism is even more complicated for the thumb [33]. Each muscle has its own moment arm and PCSA. All that information cannot be appropriately summarized in a single number. In theory, some progress could be made by making the matrix $\mathbf{K}$ depend on the sign of the torque, to distinguish between flexion and extension, or on the direction of the force produced by each finger, assuming that the end point forces are produced by clearly identified muscle synergies. An alternative approach is to extend the model to include tendons [8].

Regarding the hand posture, our results show that the effect of the subtle postural adjustments observed in the experiments did not improve the predictions. Further study will be required to know whether this result indicates that humans do not adjust hand posture in function of the orientation of the contact surfaces, or whether it is due to the coarseness of the method used to measure hand posture.

To conclude, a world of caution about the interpretation of the results of this study. Our results do not suggest that humans control contact forces solely by minimizing energy expenditure. In fact, the minimum torque model was unable to predict the changes in direction of the index and middle contact forces that contribute to the stability of the grasp by keeping the contact forces close to the normals of the contact surfaces (see Section 3). This weakness is due to the fact that the minimum torque model does not take into consideration the orientation of the contact surfaces. While this characteristic of the minimum torque model is clearly an handicap with respect to its ability to predict real grasps, it is also a prerequisite to test whether a model of 
the human grasp that minimizes energy expenditure can yield a stable grasp. More realistic models of the human grasp should include both an energy expenditure and grasp stability component.

\section{References}

1. K. An, E. Chao, and R. Linscheid W. Cooney. Forces in the normal and abnormal hand. Journal of Orthopaedic Research, 3:202-11, 1985.

2. K.N. An, Y. Ueba, E.Y. Chao, W.P. Cooney, and R.L. Linscheid. Tendon excursion and moment arm of index finger muscles. Journal of Biomechanics, 16:419-425, 1983.

3. G. Baud-Bovy and J.F. Soechting. Two virtual fingers in the control of the tripod grasp. J Neurophysiol, 86:604-615, 2001.

4. G. Baud-Bovy and J.F. Soechting. Factors influencing variability in load forces in a tripod grasp. Exp. Brain Res., 143:57-66, 2002.

5. G. Baud-Bovy and P. Viviani. Pointing to kinesthetic targets in space. J. Neuroscience, 18:1528-45, 1998.

6. N. Bernstein. Some emergent problems of the regulation of motor acts. In The coordination and regulation of movements, pages 115-142. Pergamon Press, 1967.

7. A. Bicchi. Analysis and control of power grasping. In Intelligent Robots and Systems '91. Intelligence for Mechanical Systems, Proceedings IROS '91. IEEE/RSJ International Workshop on, volume 2, pages 691-697, November 1991.

8. A. Bicchi and D. Prattichizzo. Analysis and optimization of tendinous actuation for biomorphically designed robotic systems. Robotica, 18(1):23-31, January 2000. Regular Paper.

9. M.K.O. Burstedt, J.R. Flanagan, and R.S. Johansson. Control of grasp stability in humans under different frictional conditions during multidigit manipulation. J Neurophysiol, 82:2393-2405, 1999.

10. M.R. Cutkosky. Robotic grasping and fine manipulation. Kluwer Academic Press, 1985.

11. M.R. Cutkosky and I. Kao. Computing and controlling the compliance of a robotic hand. IEEE Trans. Robotics Automat., 5(2):151-165, 1989.

12. J.R. Flanagan, M.K.O. Burstedt, and R.S. Johansson. Control of fingertip forces in multidigit manipulation. J. Neurophysiolpgy, 81:1706-1717, 1999.

13. T. Flash and N. Hogan. The coordination of arm movements: An experimentally confirmed mathematical model. J. of Neursociences, 5:1688-1703, 1985.

14. C.C.A.M. Gielen, van B.M. Bolhuis, and M. Theeuwen. On the control of biologically redundant manipulators. Human Movment Science, 14:487-509, 1995.

15. C.M. Harris and D.M. Wolpert. Signal dependent noise determines motor planning. Nature, 394:780-784, 1998.

16. T. Iberall, G. Bingham, and M.A. Arbib. Opposition space as a structuring concept for the analysis of skilled hand movements. Exp Brain Res Suppl, 10:158-173, 1986.

17. T. Iberall and C.L. Mackenzie. Opposition space and human prehension. In Dextrous Robot Hand, pages 5-31. Venkataraman ST and Iberall T. New York: Springer Verlag, 1990.

18. R.S. Johansson and J. Cole. Grasp stability during manipulative actions. Can J Physiol Pharmacol, 72:511-524, 1994.

19. K. Kim, Y. Youm, and W.K. Chung. Human kinematic factor for haptic manipulation: The wrist to thumb. Proc. of the 10th Symp. On Haptic Interfaces For Virtual Envir. and Teleoperator Systs. IEEE, 2002. 
20. J. Lee and T.L. Kunii. Model-based analysis of hand posture. IEEE Comput. Graph. Appl., 15(5):77-86, September 1995.

21. R.L. Lieber, M.D. Jacobson, B.M. Fazeli, R.A. Abrams, and M.J. Botte. Architecture of selected muscle of the arm and forearm: Anatomy and implications for tendon transfer. J. Hand Surgery, 17A(5):787-798, 1992.

22. J. Lin, Y. Wo, and T.J. Huang. Modeling the constraints of human hand motion. Proceedings. Workshop On, Human Motion:121-126, 7-8 December 2000.

23. R. L. Linscheid, K.-A. An, and R. M. Gross. Quantitative analysis of the intrinsic muscles of the hand. Clin. Anatomy, 4:265-284, 1991.

24. M.T. Mason and J.K. Salisbury. Robot hands and the mechanics of manipulation. The MIT Press, Cambridge MA, 1985.

25. R.M. Murray, Z. Li, and S.S. Sastry. A mathematical introduction to robotic manipulation. CRC, Boca Raton, Florida, 1994.

26. N. S. Pollard and R.C. Gilbert. Tendon arrangement and muscle force requirements. Conference on Robotics and Automation, Washington, D.C., May 2002.

27. D. Prattichizzo and A. Bicchi. Consistent task specification for manipulation systems with general kinematics. ASME Journal of Dynamics Systems Measurements and Control, 119:760-767, December 1997. Regular Paper.

28. D. Prattichizzo and A. Bicchi. Consistent task specification for manipulation systems with general kinematics. ASME Journal of Dynamics Systems Measurements and Control, 119:760-767, December 1997. Regular Paper.

29. D. Prattichizzo and A. Bicchi. Dynamic analysis of mobility and graspability of general manipulation systems. IEEETRA, 14(2):241-258, April 1998.

30. J.K. Salisbury and B. Roth. Kinematic and force analysis of articulated mechanical hands. J. Mech. Transm. Automat. in Des., 105, 1983.

31. M. Santello, M. Flanders, and J.F. Soechting. Postural hand synergies for tool use. J. Neuroscience, 18:10105-10115, 1998.

32. J. P. Sholz, F. Danion, M. L. Latash, and G. Shoner. Understanding finger coordination through analysis of the structure of force variability. Biol. Cybern., 86:29-39, 2002.

33. W. P. Smutz, A. Congsayreepong, R. E. Hughes, G. Niebur, W. P. Conney, and K.-N. An. Mechanical advantage of the thumb muscles. J. Biomechanics, 31:565-570, 1998.

34. J. Trinkle. On the stability and instantaneous velocity of grasped frictionless objects. IEEETRA, 8(5), 1992.

35. Y. Uno, M. Kawato, and R. Suzuki. Formation and control of optimal trajectories in human multijoint movements: Minimum torque change model. Biological Cybernetics, 61:89-101, 1989.

36. F. J. Valero-Cuevas. An integrative approach to the biomechanical function and neuromuscular control of the fingers. J. Biomechanics, In press:np, 2004.

37. F. J. Valero-Cuevas, F. E. Zajac, and C. G. Burgar. Large index-fingertip forces are produced by subject-independent patterns of muscle excitation. J. Biomechanics, 31:693703, 1998.

38. T. Yoshikawa and K. Nagai. Analysis of multi-fingered grasping and manipulation. In Dextrus Robot Hand, pages 187-208. Venkataraman ST and Iberall T. New York: Springer Verlag, 1990.

39. V.M. Zatsiorsky, F. Gao, and M.L. Latash. Finger force vectors in multi-finger prehension. Journal of Biomechanics, 36:1745-1749, 2003. 